\title{
COMPETITIVE TRAFFIC ASSIGNMENT IN ROAD NETWORKS
}

\author{
Alexander Y. Krylatov', Victor V. Zakharov', Igor G. Malygin ${ }^{2}$ \\ ${ }^{1}$ Saint Petersburg State University \\ 7/9 Universitetskaya nab., St. Petersburg, 199034 Russia \\ a.krylatov@spbu.ru,v.zaharov@spbu.ru \\ ${ }^{2}$ Solomenko Institute of Transport Problems \\ of the Russian Academy of Sciences \\ 13 12-th Line VO, St. Petersburg, 199178, Russia \\ info@iptran.ru
}

\begin{abstract}
Recently in-vehicle route guidance and information systems are rapidly developing. Such systems are expected to reduce congestion in an urban traffic area. This social benefit is believed to be reached by imposing the route choices on the network users that lead to the system optimum traffic assignment. However, guidance service could be offered by different competitive business companies. Then route choices of different mutually independent groups of users may reject traffic assignment from the system optimum state. In this paper, a game theoretic approach is shown to be very efficient to formalize competitive traffic assignment problem with various groups of users in the form of non-cooperative network game with the Nash equilibrium search. The relationships between the Wardrop's system optimum associated with the traffic assignment problem and the Nash equilibrium associated with the competitive traffic assignment problem are investigated. Moreover, some related aspects of the Nash equilibrium and the Wardrop's user equilibrium assignments are also discussed.
\end{abstract}

Keywords: competitive traffic assignment, Nash equilibrium, system optimum of Wardrop, user equilibrium of Wardrop

\section{Introduction}

The rapid development of information technologies in the past three decades has led to the emergence of different specialized telecommunication systems. Nowadays these systems are introduced almost in every field of human activity including transportation. Without shadow of doubt, the influence of these systems on decision making increases significantly. The impact of in-vehicle route guidance and information (IVRGI) systems on route choice one could observe in his/her own daily trips. Moreover, the permanent innovative development of such systems is noticeably related to the creation of intelligent vehicles. Indeed, from a consumer perspective one of the main attributes of any intelligent vehicle is an automatic drive regime that is associated with an automatic in-vehicle route guidance system. Therefore, guidance systems are seemed to be an integral part of the concept of intelligent vehicle.

Actually, an automatic guidance system is a great advantage of intelligent vehicles not only from a consumer perspective. The traffic flow of intelligent vehicles could be automatically assigned by the central guidance system in the way to minimize overall travel time of all road network users. In other words, a system optimum (Wardrop, 1952) could be reached on the network by imposing the optimal route choices on the users (Patriksson, 2015; Sheffi, 1985). Such an assignment of traffic flow is often called involuntary system optimum, unlike voluntary system optimum (Gartner, 1980). In a voluntary system optimum case, after paying special charging tolls users reached system optimum, although initially they were tending to user equilibrium assignment (Patriksson, 2015; Sheffi, 1985). Here, it should be mentioned that user equilibrium assignment is supposed to take place when all drivers tries to minimize their own travel time without support of any guidance system. As a result, the overall travel time spending by all users assigned according to the interest of each atomic user is more than overall travel time in the system optimum case. Therefore, the central guidance system is capable to reduce congestions by imposing system optimum assignment on the intelligent vehicles.

Moreover, according to Bonsall (1992) there is considerable government interest in the development of in-vehicle guidance systems. This interest reflects a belief that such systems could produce benefits in four ways (Bonsall, 1992):

- to improve people's knowledge of the network and assist them to find efficient routes;

- to reduce unnecessary mileage, traffic volumes, and hence congestion;

- to link in-vehicle guidance system with traffic control and, perhaps, road pricing systems;

- to obtain more globally efficient routing patterns (system optimum). 
Therefore governments feel possible positive effects from implementation of in-vehicle guidance systems and try to formalize them. From the mathematical side all these advantages could be expressed by the system optimum principle. Hence, all the mentioned ways of producing benefits to the traffic systems are already discussed in the previous paragraph in a short form of the "optimizational vocabulary".

Despite the interest of government, as a rule the major contribution in development of guidance systems are produced by different private business companies. By the virtue of competitive structure of economics these companies are forced to compete with each other offering their own users better service. First of all, "better service" means the less travel time from origin to destination point. Thus, each company seeks to route the flows of its own users so to minimize their average travel time. At the same time the others try to minimize the average travel time of their users routing in the same road network. Due to the described circumstances the competitive traffic assignment problem has appeared. Noncooperative nature of relations between the companies leads to the set of such optimization programs that the unknown variables of any of these programs are independent parameters in all others. Therefore, competitive traffic assignment problem should be formulated in a game theoretic form with Nash equilibrium search (Nash, 1951).

The system optimum assignment obtained by in-vehicle guidance systems with one provider is assumed to differ from the assignment imposed by Nash equilibrium strategies of the competitive companies offered their consumers route guidance. Thus, the investigation of relationships between Wardrop's system optimum associated with the traffic assignment problem and Nash equilibrium associated with competitive traffic assignment problem seems quite important. Indeed, when flows of intelligent vehicles are large enough then competitive guidance systems could deviate traffic assignment from system optimum significantly. This fact should be taken into consideration by traffic engineers, transportation planners, network designers and etc. in transportation modelling. This paper is completely focused on the mentioned relationships and moreover, some common aspects of Nash equilibrium and user equilibrium of Wardrop assignments will be also illuminated.

The paper is organized as follows. Section II is devoted to the discussion of such research direction as competitive traffic assignment that is found in this paper. The brief analysis of the existing papers that have any formal similarities with the competitive traffic assignment problem is carried out in Section III. In Section IV the mathematical formulation of traffic assignment problem on the network of general topology is introduced. Competitive behaviour of different groups of users is formulated in a game theoretic form in Section V. Section VI is addressed to the results concerning relationships between Nash equilibrium assignment and Wardrop's assignment. Conclusions for this paper are briefly summarized in Section VII.

\section{Discussion of the research direction}

There already exists a huge amount of researches devoted to Wardrop's principles of traffic assignment. The extensive review of the existing models and methods implemented for traffic assignment evaluation is made by M. Patriksson in 1994 (Patriksson, 1994). This outstanding book was republished in the beginning of 2015 without loss of its relevance (Patriksson, 2015). In spite of various results already obtained in this branch of applied mathematics, new investigations are still appearing to contribute namely practically expanding numerical techniques. For instance, in the past decade much attention has been paid to a class of bushbased algorithms (Zheng and Peeta, 2014). According to Xie et al. (2013), it is the ability of such algorithms to solve large-scale traffic assignment problems at a high level of precision that attracts many researchers. On the other hand, there has been a relatively weak development of theoretical principles concerning traffic assignment problem since the early 1950s. Indeed, the first principle of Wardrop associated with the user equilibrium assignment states:

- The journey times on all routes actually used are equal, and less than those which would be experienced by a single vehicle on any unused route.

This principle cited from Wardrop's article (1952) is still relevant and useful for evaluation of traffic flow assignment. In other words, traffic engineers and decision makers at the different levels of management use this principle to identify the used routes in the network and their flows. Another principle of Wardrop associated with system optimum and offered in the same article states:

- The average journey time is a minimum.

This principle could not be used for evaluation of traffic flow assignment since no one driver seeks to minimize average time. Any driver tries to minimize its own travel time and, hence, the first principle of Wardrop is more appropriate for the purpose of such evaluation. Nevertheless, the relationships between the user equilibrium and the system optimum define important economical conclusions in transportation 
about toll pricing on the links of network (Gartner, 1980). Eventually, due to the second Wardrop's principle it is possible to evaluate toll prices that could be charged from users of a road network.

Therefore, the both Wardrop's principles are useful from practical perspective. However, the drawback is that they are not applicable when instead of atomic drivers the behaviour of groups of drivers (with common group interest) should be taken into consideration. Thus, by virtue of rapid guidance systems development the need for a new assignment principle is expected to increase significantly in the coming years. If the term "group of users (UG)" we understand as a set of all drivers following advices of the same guidance system, then a new principle could be formulated roughly as follows:

- Under competitive conditions the average journey time of each group of users there is a minimum.

Note, the explicit mention of the competitive environments in this principle is important since without competitive behaviour of companies creating guidance systems the second principle of Wardrop is sufficient for the purpose of appropriate modelling. Further we will associate this competitive traffic assignment principle with the competitive traffic assignment problem.

\section{State-of-the-art}

The first attempt to define traffic equilibria in forms of network games was made in the late 1950s by Charnes and Cooper (1958). They described the user equilibrium flow as a non-cooperative Nash equilibrium in a game where the players are pairs of origin-destination (OD pairs), competing to minimize travel time of their respective commodity flows. Further discussion along this line is developed by Dafermos (1971) and Dafermos and Sparrow (1969). However, these first investigations of the relationships between a Wardrop equilibrium and a network games have not driven to any formal expressions.

Rosenthal studied a discrete version of the user equilibrium traffic assignment problem (Rosenthal, 1973). It should be stressed that the players are defined as the individual travellers, with strategy spaces equal to their respective sets of routes available. Travellers seeking to minimize their individual travel time, i.e. their payoff functions. The game is shown to be equivalent to a non-cooperative, pure-strategy Nash game in the traffic network. Therefore, he was the first one who formulated a special case of competitive traffic assignment problem as we defined it above. This is the special case since each UG consist of solely one user.

Devarajan in 1981 extended discrete version to the continuous case, however, as do Charnes and Cooper, defines OD pairs as the players (Devarajan, 1981) and consider the payoff functions:

$$
\varphi_{\omega}(\mathbf{y})=\sum_{a \in A_{\omega}} \int_{0}^{y_{a}} t_{a}(s) d s, \quad \forall \omega \in W,
$$

where $W$ is a set of UGs, $\omega \in W ; A_{\omega}$ is a set of links included in routes between OD pair $\omega ; y_{a}$ is a traffic flow on a congested link $a ; t_{a}$ is a travel time through a congested link $a$. Hence this formulation does not correspond to competitive traffic assignment principle. Nevertheless, it was proved that the Nash game thus defined is equivalent to a Wardrop equilibrium.

In the middle of 1980s more general game formulations of traffic equilibria were given by Fisk (1984) and Haurie and Marcotte (1985). The travel made in an OD pair is divided into a number of players and, hence, a player, as defined, may use several routes simultaneously; in equilibrium, all players divide their flow on all routes used in the OD pair. Therefore, such formulation has the certain common features with competitive traffic assignment problem. However, it is shown that only in the limiting case, when the number of players in each OD pair tends to infinity, while sharing the same strategy, the Nash game is equivalent to a Wardrop equilibrium.

In 1990 the development of computer networks motivated researchers to begin investigation of competitive routing in multiuser communication networks (Orda et al., 1993). According to Orda et al., a single administrative domain was no longer a valid assumption in networking. Then communication networks shared by selfish users with their own given flow demands were considered and modelled as noncooperative games by several research groups (Korilis and Lazar, 1995; Korilis et al., 1995; La and Anantharam, 1997; Altman et al., 2002). Due to these researches different properties of such systems are established, and the conditions of existence and uniqueness of Nash equilibrium in multiuser communication networks are widely studied. 
During the 2000s Altman et al. have extended results, obtained for multiuser communication networks, and then implemented them to road networks (Altman et al., 2002; Altman and Kameda, 2005; Altman et al., 2011). Unlike Haurie and Marcotte, Altman et al. established the convergence of the Nash equilibrium in network games to the Wardrop equilibrium as the number of players grows under weaker convexity assumptions (Altman et al., 2011). Therefore, they raised again the question of relationships between Nash equilibrium in noncooperative n-person network games and Wardrop equilibrium in the traffic assignment problem.

\section{Traffic assignment problem}

Consider a transportation network presented by oriented graph $G=(N, A)$. We assume, that there is a set of OD pairs $W$ and the sets of routes $K_{\omega}$ between each OD pair $\omega \in W$. Moreover, introduce following notation: $F^{\omega}$ is the demand between $\omega ; f_{k}^{\omega}$ is the flow of UG $j$ through $k \in K_{\omega} ; x_{a}$ is the flow through the arc $a \in A, x=\left(\ldots, x_{a}, \ldots\right) ; t_{a}(x)=t_{a}\left(x_{a}\right)$ is the travel time of flow $x_{a}$ through congested arc $a \in A ; \delta_{a, k}^{j \omega}$ is an indicator: 1 if UG $j$ uses route $k \in K_{\omega}$, that "includes" arc $a \in A$, and 0 otherwise.

\subsection{User equilibrium assignment}

According to (Beckmann, 1956; Sheffi, 1985; Patriksson, 1994), the equal travel time on all actually used routes, that is less than travel time on any unused route, could be reached by assignment strategy obtained from the following optimization program:

$$
\mathbf{Z}^{u e}\left(\mathbf{x}^{u e}\right)=\min _{x} \mathbf{Z}^{u e}(x)=\min _{x} \sum_{a \in A} \int_{0}^{x_{a}} t_{a}(u) d u,
$$

subject to

$$
\begin{aligned}
& \sum_{k \in K_{\omega}} f_{k}^{\omega}=F^{\omega} \quad \forall \omega \in W, \\
& f_{k}^{\omega} \geq 0 \quad \forall k \in K_{\omega}, \omega \in W,
\end{aligned}
$$

with definitional constraints

$$
x_{a}=\sum_{\omega \in W} \sum_{k \in K_{\omega}} f_{k}^{\omega} \delta_{a, k}^{\omega} \quad \forall a \in A .
$$

\subsection{System optimum assignment}

According to (Beckmann, 1956; Sheffi, 1985; Patriksson, 1994), the minimum average travel time could be reached by assignment strategy obtained from the following optimization program:

$$
\mathbf{Z}^{\text {so }}\left(\mathbf{x}^{\text {so }}\right)=\min _{x} \mathbf{Z}^{\text {so }}(x)=\min _{x} \sum_{a \in A} t_{a}\left(x_{a}\right) x_{a},
$$

subject to

$$
\begin{aligned}
& \sum_{k \in K_{\omega}} f_{k}^{\omega}=F^{\omega} \quad \forall \omega \in W, \\
& f_{k}^{\omega} \geq 0 \quad \forall k \in K_{\omega}, \omega \in W,
\end{aligned}
$$

with definitional constraints

$$
x_{a}=\sum_{\omega \in W} \sum_{k \in K_{\omega}} f_{k}^{\omega} \delta_{a, k}^{\omega} \quad \forall a \in A .
$$




\section{Competitive traffic assignment problem}

Consider the same network presented by oriented graph $G=(N, A)$, set of OD pairs $W$ and corresponding sets of routes $K_{\omega}, \omega \in W$. According to competitive traffic assignment principle, each group tries to assign its users among available routes from origin to destination in such a way, that their average travel time is minimum. Introduce following notation: $M=1, \ldots, m\}$ is the set of users' groups (UG); $F^{j \omega}>0$ is the demand of UG $j$ between $\omega, F^{j}=\sum_{\omega \in W} F^{j \omega} ; x_{a}^{j}$ is the flow of UG $j$ through the $\operatorname{arc} a \in A, x^{j}=\left(\ldots, x_{a}^{j}, \ldots\right), x^{-j}=\left(x_{1}, \ldots, x_{a}^{j-1}, x_{a}^{j+1}, \ldots, x_{m}\right)$ and $x_{a}=\left(x_{a}^{1}, \ldots, x_{a}^{m}\right) ; f_{k}^{j \omega}$ is the flow of UG $j$ through $k \in K_{\omega} ; f^{j \omega}=\left(f_{1}^{j \omega}, \ldots, f_{\left|K_{\omega}\right|}^{j \omega}\right)^{\mathrm{T}}$ is the assignment of the flow $F^{j \omega}$ through possible routes $K_{\omega} ; f^{j}=\left(\ldots, f^{j \omega}, \ldots\right)$ is the strategy of $\mathrm{UG} j$ (the assignment of the flows $F^{j \omega}$ between all OD-pairs), and $f^{-j}=\left(f^{1}, \ldots, f^{j-1}, f^{j+1}, \ldots, f^{m}\right) ; f=\left(f^{1}, \ldots, f^{m}\right)$ is the set of all strategies of all UGs.

Each UG tries to minimize the average travel time of its own users. Therefore, the following optimization programs could be formulated for all $j=\overline{1, m}$ (Zakharov and Krylatov, 2014, 2016):

$\mathbf{Z}_{m}^{j}\left(\mathbf{x}^{j^{*}}, x^{-j}\right)=\min _{x^{j}} \mathbf{Z}_{m}^{j}(x)=\min _{x^{j}} \sum_{a \in A} t_{a}\left(x_{a}\right) x_{a}^{j}$,

subject to

$$
\begin{aligned}
& \sum_{k \in K_{\omega}} f_{k}^{j \omega}=F^{j \omega} \quad \forall \omega \in W, \\
& f_{k}^{j \omega} \geq 0 \quad \forall k \in K_{\omega}, \omega \in W,
\end{aligned}
$$

with definitional constraints

$$
\begin{aligned}
& x_{a}^{j}=\sum_{\omega \in W k \in K_{\omega}} \sum_{k}^{j \omega} \delta_{a, k}^{j \omega} \quad \forall a \in A . \\
& x_{a}=\sum_{j=1}^{m} x_{a}^{j} \quad \forall a \in A .
\end{aligned}
$$

Note, that for each $j \in M$ the set $x^{-j}$ is not fixed, but induced by the assignment decisions of other UGs. Therefore, we obtain competitive traffic assignment problem, that could be reformulated in a form of noncooperative network game with penalty functions $Z^{j}, \quad j=\overline{1, m}$ : $\boldsymbol{\Gamma}_{m}\left(M,\left\{\mathbf{F}_{m}^{j}\right\}_{j \in M},\left\{\mathbf{Z}_{m}^{j}\right\}_{j \in M}\right)$, where $\mathbf{F}_{m}^{j}=\left\{f^{j \omega} \mid f_{k}^{j \omega} \geq 0 \forall k \in K_{\omega}, \sum_{k \in K_{\omega}} f_{k}^{j \omega}=F^{j \omega} \forall \omega \in W\right\}$, when

$$
x_{a}^{j}=\sum_{\omega \in W} \sum_{k \in K_{\omega}} f_{k}^{j \omega} \delta_{a, k}^{j \omega} \quad \text { and } \quad x_{a}=\sum_{j=1}^{m} x_{a}^{j} .
$$

Consideration of competitive traffic assignment problem in a game theoretic form leads us to the Nash equilibrium search. Nash equilibrium in the game $\boldsymbol{\Gamma}_{m}$ is realized by strategies $\mathbf{x}_{m}^{n e}=\left(\mathbf{x}^{1^{*}}, \ldots, \mathbf{x}^{m^{*}}\right)$ such that

$$
\mathbf{Z}_{m}^{j}\left(\mathbf{x}_{m}^{n e}\right) \leq \mathbf{Z}_{m}^{j}\left(x^{j}, \mathbf{x}^{-j^{*}}\right) \quad \forall j \in M .
$$




\section{Relationships between competitive and non-competitive traffic assignments}

There are certain relationships between optimization programs (1)-(4), (5)-(8) and (9)-(13). These relationships are defined by the following lemma.

Lemma 1. Ceteris paribus it is true, that

1) the system optimum goal function is equal to the sum of goal functions of all UGs:

$$
\mathbf{Z}^{s o}(x)=\sum_{j \in M} \mathbf{Z}_{m}^{j}(x) \quad \forall x, \forall m \geq 1,
$$

2) competitive atomic and group behaviour of vehicles deviate transportation system from optimal state:

$\mathbf{Z}^{\text {so }}\left(\mathbf{x}^{\text {so }}\right)\left\{\begin{array}{l}\leq \mathbf{Z}^{\text {so }}\left(\mathbf{x}^{\text {ue }}\right) \text { in an atomic case, } \\ \leq \mathbf{Z}^{\text {so }}\left(\mathbf{x}_{m}^{n e}\right) \text { in a group case, }\end{array}\right.$

particularly, if $t_{a}\left(x_{a}\right) \neq$ const, then there are strict inequalities:

$\mathbf{Z}^{\text {so }}\left(\mathbf{x}^{\text {so }}\right)\left\{\begin{array}{l}<\mathbf{Z}^{\text {so }}\left(\mathbf{x}^{u e}\right) \text { in an atomic case }, \\ <\mathbf{Z}^{\text {so }}\left(\mathbf{x}_{m}^{\text {ne }}\right) \text { in a group case. }\end{array}\right.$

Proof. 1) Consider the sum of goal functions of all UGs:

$$
\sum_{j=1}^{m} \mathbf{Z}_{m}^{j}(x)=\sum_{j=1}^{m} \sum_{a \in A} t_{a}\left(x_{a}\right) x_{a}^{j}=\sum_{a \in A} t_{a}\left(x_{a}\right)\left(\sum_{j=1}^{m} x_{a}^{j}\right)=\sum_{a \in A} t_{a}\left(x_{a}\right) x_{a}=\mathbf{Z}^{s o}(x) .
$$

2) Since $\mathbf{x}^{\text {so }}$ is the global minimum of $\mathbf{Z}^{\text {so }}$, then $\mathbf{Z}^{\text {so }}\left(\mathbf{x}^{\text {so }}\right) \leq \mathbf{Z}^{\text {so }}(x)$ for any possible $X$, and, particularly for $\mathbf{x}^{\text {ue }}$ and $\mathbf{x}_{m}^{n e}$.

3a) It is well known that system optimum is equal to user equilibrium only if travel times $t_{a}\left(x_{a}\right)$ do not depend on the value of flow (Patriksson, 2015). Indeed, $\mathbf{x}^{\text {ue }}$ satisfies an equality:

$\sum_{a \in A} t_{a}\left(\mathbf{x}^{u e}\right) \delta_{a, k}^{\omega}=T_{\omega}^{u e} \quad \forall k \in K_{\omega}, \omega \in W$,

where $T_{\omega}^{u e}$ is an optimal travel time through any route between $\omega$ OD-pair in an user equilibrium state. On the other hand, $\mathbf{x}^{\text {so }}$ satisfies another equality:

$$
\sum_{a \in A}\left[t_{a}\left(\mathbf{x}^{s o}\right)+\frac{\partial t_{a}\left(\mathbf{x}^{s o}\right)}{\partial x_{a}}\right] \delta_{a, k}^{\omega}=T_{\omega}^{s o} \quad \forall k \in K_{\omega}, \omega \in W,
$$

where $T_{\omega}^{s o}$ is an optimal marginal cost of any route between $\omega$ OD-pair in a system optimum state. Expression (18) could be equal to (19) only if

$$
\sum_{a \in A} \frac{\partial t_{a}\left(\mathbf{x}^{s o}\right)}{\partial x_{a}} \delta_{a, k}^{\omega}=0 \quad \forall k \in K_{\omega}, \omega \in W,
$$

that means $t_{a}\left(x_{a}\right)=$ const for all $a \in A$.

3b) To prove that $\mathbf{Z}^{\text {so }}\left(\mathbf{x}^{\text {so }}\right)<\mathbf{Z}^{\text {so }}\left(\mathbf{x}_{m}^{\text {ne }}\right)$ when $t_{a}\left(x_{a}\right) \neq$ const, let us consider the Lagrangian of optimization program associated with any UG $j=\overline{1, m}$ and expressed by (9)-(13): 


$$
L^{j}=\sum_{a \in A} t_{a}\left(x_{a}\right) x_{a}^{j}+T_{\omega}^{j, m}\left(F^{j \omega}-\sum_{k \in K_{\omega}} f_{k}^{j \omega}\right)+\sum_{k \in K_{\omega}}\left(-f_{k}^{j \omega}\right) \eta_{k}^{j \omega},
$$

where $T_{\omega}^{j, m}$ and $\eta_{k}^{j \omega} \geq 0$ are Lagrange multipliers. According to Kuhn-Tucker conditions (for optimal $f_{k}^{j \omega}>0$ ) we obtain

$$
\frac{\partial L^{j}}{\partial x_{a}^{j}}=\sum_{a \in A}\left[t_{a}\left(x_{a}\right)+\frac{\partial t_{a}\left(x_{a}\right)}{\partial x_{a}^{j}} x_{a}^{j}\right]-T_{\omega}^{j, m} \delta_{a, k}^{j \omega}=0
$$

and consequently

$$
\sum_{a \in A}\left[t_{a}\left(\mathbf{x}_{m}^{n e}\right)+\frac{\partial t_{a}\left(\mathbf{x}_{m}^{n e}\right)}{\partial x_{a}^{j}} x_{a}^{j^{*}}\right] \delta_{a, k}^{j \omega}=T_{\omega}^{j, m} .
$$

Moreover, since $x_{a}=\sum_{j=1}^{m} x_{a}^{j}$ then $\frac{\partial x_{a}}{\partial x_{a}^{j}}=1$ and, hence,

$$
\frac{\partial t_{a}\left(x_{a}\right)}{\partial x_{a}^{j}}=\frac{\partial t_{a}\left(x_{a}\right)}{\partial x_{a}} \cdot \frac{\partial x_{a}}{\partial x_{a}^{j}}=\frac{\partial t_{a}\left(x_{a}\right)}{\partial x_{a}} \text {. }
$$

Therefore, (20) could be rewritten as

$$
\sum_{a \in A}\left[t_{a}\left(\mathbf{x}_{m}^{n e}\right)+\frac{\partial t_{a}\left(\mathbf{x}_{m}^{n e}\right)}{\partial x_{a}} x_{a}^{j^{*}}\right] \delta_{a, k}^{j \omega}=T_{\omega}^{j, m} \quad \forall j=\overline{1, m} .
$$

Eventually, expression (18) could be equal to (19) only if

$$
\sum_{a \in A} \frac{\partial t_{a}\left(\mathbf{x}_{m}^{n e}\right)}{\partial x_{a}} \delta_{a, k}^{\omega}=0 \quad \forall k \in K_{\omega}, \omega \in W
$$

that means $t_{a}\left(x_{a}\right)=$ const for all $a \in A$.

Lemma is proved.

Remark. Since in real road networks travel time significantly depend on congestion one could believe that competitive atomic and group behaviour of vehicles strictly increases the average travel time.

Unfortunately, Lemma 1 does not give any answer to the question about ratio of $\mathbf{Z}^{\text {so }}\left(\mathbf{x}^{\text {ue }}\right)$ and $\mathbf{Z}^{\text {so }}\left(\mathbf{x}_{m}^{n e}\right)$. The only fact we obtained is that $\mathbf{Z}^{\text {so }}\left(\mathbf{x}^{u e}\right)$ and $\mathbf{Z}^{\text {so }}\left(\mathbf{x}_{m}^{n e}\right)$ are both larger then $\mathbf{Z}^{\text {so }}\left(\mathbf{x}^{u e}\right)$ and $\mathbf{Z}^{\text {so }}\left(\mathbf{x}^{\text {so }}\right)$, i.e. atomic and group behavior of drivers increase the average travel time in a road network.

Theorem 1. The following inequalities hold

$$
\mathbf{Z}^{s o}\left(\mathbf{x}^{s o}\right) \leq \mathbf{Z}^{s o}\left(\mathbf{x}_{m}^{n e}\right) \leq \mathbf{Z}^{\text {so }}\left(\mathbf{x}^{u e}\right) .
$$
part of (22).

Proof. Left part of (22) is proved by previous Lemma. Let us concentrate on the proof of the right

First of all, we should compare network games $\boldsymbol{\Gamma}_{m}$ and $\boldsymbol{\Gamma}_{m+1}$. According to Lemma 1 $\mathbf{Z}^{\text {so }}(x)=\sum_{j \in M} \mathbf{Z}_{m}^{j}(x)$ and this is true for any amount of UGs. Therefore,

$$
\mathbf{Z}^{s o}(x)=\sum_{j=1}^{m} \mathbf{Z}_{m}^{j}(x)=\sum_{j=1}^{m+1} \mathbf{Z}_{m+1}^{j}(x) \quad \forall x
$$


The sets of constraints of games $\boldsymbol{\Gamma}_{m}$ and $\boldsymbol{\Gamma}_{m+1}$ satisfy the inclusion $\bigcup_{j=1}^{m} \mathbf{F}_{m}^{j} \subseteq \bigcup_{j=1}^{m+1} \mathbf{F}_{m+1}^{j}$. Subsequently, we have the inequality

$\mathbf{Z}^{\text {so }}\left(\mathbf{x}_{m}^{\text {ne }}\right) \leq \mathbf{Z}^{\text {so }}\left(\mathbf{x}_{m+1}^{\text {ne }}\right)$.

Note that in real road network $|F|$ (the absolute amount of all vehicles) is a maximum amount of UGs. In such a case each atomic vehicle tries to minimize its own travel time. Formally, in this situation $m$ and $F^{j}$ could be expressed as $m=|F|$ and $F^{j}=1$ for all $j=\overline{1,|F|}$. Hence, due to (24) we obtain

$$
\mathbf{Z}^{s o}\left(\mathbf{x}_{m}^{n e}\right) \leq \mathbf{Z}^{s o}\left(\mathbf{x}_{|F|}^{n e}\right) \quad \forall m=\overline{1,|F|} .
$$

On the other hand, the set of strategies in the network game $\boldsymbol{\Gamma}_{|F|}$ is a Nash equilibrium if no vehicle can do better by unilaterally changing their route. However, this is a Wardrop equilibrium by definition. Therefore, Nash equilibrium in network game $\boldsymbol{\Gamma}_{|F|}$ and it should be equal to Wardrop equilibrium in the same network. Nevertheless, a certain clarification should be made.

Consider optimization program (1)-(4) associated with user equilibrium of Wardrop. The solution of this program satisfies (18) and, without a doubt, generally it is not integer. Therefore, if we find Nash equilibrium in network game $\boldsymbol{\Gamma}_{|F|}$ in pure strategies, then Nash equilibrium is not equal to Wardrop equilibrium: $\mathbf{Z}^{\text {so }}\left(\mathbf{x}_{|F|}^{n e}\right) \leq \mathbf{Z}^{\text {so }}\left(\mathbf{x}^{u e}\right)$. Nevertheless, if we require $x$ to be integer in (1)-(4) and call the solution integer user equilibrium of Wardrop, then Nash equilibrium in pure strategies is equal to integer Wardrop equilibrium: $\mathbf{Z}^{\text {so }}\left(\mathbf{x}_{|F|}^{\text {ne }}\right)=\mathbf{Z}^{\text {so }}\left(\mathbf{x}^{\text {ue }}\right)$. Eventually, if we find Nash equilibrium in $\boldsymbol{\Gamma}_{|F|}$ in mixed strategies, then Nash equilibrium in mixed strategies is equal to Wardrop equilibrium: $\mathbf{Z}^{\text {so }}\left(\mathbf{x}_{|F|}^{\text {ne }}\right)=\mathbf{Z}^{\text {so }}\left(\mathbf{x}^{\text {ue }}\right)$. Therefore, depending on the additional conditions:

$\mathbf{Z}^{\text {so }}\left(\mathbf{x}_{|F|}^{\text {ne }}\right)=\mathbf{Z}^{\text {so }}\left(\mathbf{x}^{u e}\right)$ or $\mathbf{Z}^{\text {so }}\left(\mathbf{x}_{|F|}^{\text {ne }}\right)<\mathbf{Z}^{\text {so }}\left(\mathbf{x}^{u e}\right)$.

Theorem is proved.

Corollary 1. If $t_{a}\left(x_{a}\right) \neq$ const then the following inequalities hold

$$
\mathbf{Z}^{\text {so }}\left(\mathbf{x}^{\text {so }}\right)=\mathbf{Z}^{\text {so }}\left(\mathbf{x}_{1}^{\text {ne }}\right)<\mathbf{Z}^{\text {so }}\left(\mathbf{x}_{2}^{\text {ne }}\right)<\ldots<\mathbf{Z}^{\text {so }}\left(\mathbf{x}_{m}^{\text {ne }}\right)<\mathbf{Z}^{\text {so }}\left(\mathbf{x}_{m+1}^{\text {ne }}\right)<\ldots<\mathbf{Z}^{s o}\left(\mathbf{x}_{|F|}^{\text {ne }}\right) \leq \mathbf{Z}^{\text {so }}\left(\mathbf{x}^{\text {ue }}\right) .
$$

Proof. By virtue of (21):

- for $m$ UGs the following $m$ equations hold, $j=\overline{1, m}$ :

$$
\sum_{a \in A}\left[t_{a}\left(\mathbf{x}_{m}^{n e}\right)+\frac{\partial t_{a}\left(\mathbf{x}_{m}^{n e}\right)}{\partial x_{a}} x_{a}^{j *}\right] \delta_{a, k}^{j \omega}=T_{\omega}^{j, m}
$$

- for $m+1$ UGs the following $m+1$ equations hold, $j=\overline{1, m+1}$ :

$$
\sum_{a \in A}\left[t_{a}\left(\mathbf{x}_{m+1}^{n e}\right)+\frac{\partial t_{a}\left(\mathbf{x}_{m+1}^{n e}\right)}{\partial x_{a}} x_{a}^{j *}\right] \delta_{a, k}^{j \omega}=T_{\omega}^{j, m+1} .
$$

Therefore, $\mathbf{x}_{m}^{\text {ne }}$ and $\mathbf{x}_{m+1}^{\text {ne }}$ could be equal only if $t_{a}\left(x_{a}\right)=$ const, then due to (24) we obtain (26).

Corollary is proved.

Obtained results testify that, in the case of competing UGs on the transportation network, the average travel time of traffic flows between origin and destination areas possibly increases in comparison 
with an existing of only one UG. In other words, competitive using of network leads to appearance of some increment in average travel time.

\section{Conclusions}

The proof of Theorem 1 allows us to establish some rules that characterize the border conversion of competitive assignment (Nash equilibrium) into non-competitive assignment (user equilibrium and system optimum). Here they are:

- if $j=1$ then Nash-equilibrium is converted into system optimum of Wardrop,

- if $F^{j}=1$ for all $j=\overline{1, m}$ then Nash equilibrium in pure strategies is converted into integer user equilibrium of Wardrop,

- if $F^{j}=1$ for all $j=\overline{1, m}$ then Nash equilibrium in mixed strategies is converted into user equilibrium of Wardrop,

- if $F^{j} \rightarrow 1$ for all $j=\overline{1, m}$ or equivalently $m \rightarrow \infty$ then Nash equilibrium is converted into user equilibrium of Wardrop.

Moreover, due to Corollary one could state three important conclusions:

1) Competitive in-vehicle route guidance systems decrease the average travel time in urban traffic area in comparison with an atomic vehicle guidance.

2) The fewer amounts of competitive in-vehicle guidance systems, the less average travel time in urban traffic area.

3) Centralized guidance system guarantees the least travel time in urban traffic area.

Therefore, since in modern worldwide cities drivers chose routes by their own even competitive guidance systems could decrease the average travel time. Consequently, from this perspective, the development of intelligent vehicles could significantly improve traffic conditions in urban areas.

\section{References}

1. Altman, E., Basar, T., Jimenez, T., Shimkin, N. (2002) Competitive routing in networks with polynomial costs. IEEE Transactions on automatic control, 47(1), 92-96.

2. Altman, E., Combes, R., Altman, Z., Sorin, S. (2011) Routing games in the many players regime. In: Proceedings of the 5th International ICST Conference on Performance Evaluation Methodologies and Tools, pp. 525-527.

3. Altman, E., Kameda, H. (2005) Equilibria for multiclass routing problems in multi-agent networks. Advances in Dynamic Games, 7, 343- 367.

4. Beckmann, M.J., McGuire, C.B., Winsten, C.B. (1956) Studies in the Economics of Transportation. New Haven, CT: Yale University Press.

5. Bonsall, P. (1992) The influence of route guidance advice on route choice in urban networks. Transportation, 19, 1-23.

6. Charnes, A., Cooper, W.W. (1958) Extremal principles for simulating traffic flow in a network. Proceedings of the National Academy of Science of the United States of America, 44, 201-204.

7. Dafermos, S.C. (1971) An extended traffic assignment model with applications to two-way traffic. Transportation Science, 5, 366-389.

8. Dafermos, S.C., Sparrow, F.T. (1969) The traffic assignment problem for a general network. Journal of Research of the National Bureau of Standards, 73B, 91-118.

9. Devarajan, S. (1981) A note on network equilibrium and noncooperative games. Transportation Research, 15B, 421-426.

10. Fisk, C.S. (1984) Game theory and transportation systems modelling. Transportation Research, 18B, 301-313.

11. Gartner, N.H. (1980) Optimal traffic assignment with elastic demands: a review. Part I. Analysis framework. Transportation Science, 14(2), 174-191.

12. Haurie, A., Marcotte, P. (1985) On the relationship between Nash-Cournot and Wardrop equilibria. Networks, 15, 295-308.

13. Korilis, Y.A., Lazar, A.A. (1995) On the existence of equilibria in noncooperative optimal flow control. Journal of the Association for Computing Machinery, 42(3), 584-613.

14. Korilis, Y.A., Lazar, A.A., Orda, A. (1995) Architecting noncooperative networks. IEEE J. Selected Areas Commun., 13, 1241-1251. 
15. La, R.J., Anantharam, V. (1997) Optimal routing control: game theoretic approach. In: Proc. of the 36th IEEE Conference on Decision and Control, 2910-2915.

16. Nash, J. (1951) Non-cooperative games. Annals of Mathematics, 54, 286-295.

17. Orda, A., Rom, R., Shimkin, N. (1993) Competitive routing in multiuser communication networks. IEEE/ACM Transactions on Networking, 1(5), 510-521.

18. Patriksson, M. (1994) The traffic assignment problem: models and methods. Utrecht, Netherlands: VSP Publishers.

19. Patriksson, M. (2015) The traffic assignment problem: models and methods. N.Y., USA: Dover Publications, Inc.

20. Rosenthal, R.W. (1973) The network equilibrium problem in integers. Networks, 3, 53-59.

21. Sheffi, Y. (1985) Urban transportation networks: equilibrium analysis with mathematical programming methods. N.J.: Prentice-Hall, Inc, Englewood Cliffs.

22. Wardrop, J.G. (1952) Some theoretical aspects of road traffic research. Proc. Institution of Civil Engineers, 2, 325-378.

23. Xie, J., Yu, N., Yang, X. (2013) Quadratic approximation and convergence of some bush-based algorithms for the traffic assignment problem. Transportation research Part B, 56, 15-30.

24. Zakharov, V., Krylatov, A. (2014) Equilibrium Assignments in Competitive and Cooperative Traffic Flow Routing. IFIP Advances in Information and Communication Technology, 434, 641-648.

25. Zakharov, V., Krylatov, A. (2016) Competitive routing of traffic flows by navigation providers. Automation and Remote Control, 77(1), 179-189.

26. Zheng, H., Peeta, S. (2014) Cost scaling based successive approximation algorithm for the traffic assignment problem. Transportation research Part B, 68, 17-30. 